\title{
THE
}

2005

\section{Observations of Steep Wave Statistics in Open Ocean Waters}

\author{
Nicholas Scott \\ University of Rhode Island \\ Tetsu Hara \\ University of Rhode Island, thara@uri.edu
}

Edward J. Walsh

Paul A. Hwang

Follow this and additional works at: https://digitalcommons.uri.edu/gsofacpubs

\section{Citation/Publisher Attribution}

Scott, N., Hara, T., Walsh, E. J., \& Hwang, P. A. (2005). Observations of Steep Wave Statistics in Open Ocean Waters. Journal of Atmospheric and Oceanic Technology, 22, 258-271.doi: 10.1175/JTECH1702.1 Available at: https://doi.org/10.1175/JTECH1702.1

This Article is brought to you for free and open access by the Graduate School of Oceanography at DigitalCommons@URI. It has been accepted for inclusion in Graduate School of Oceanography Faculty Publications by an authorized administrator of DigitalCommons@URI. For more information, please contact digitalcommons-group@uri.edu. 


\title{
Observations of Steep Wave Statistics in Open Ocean Waters
}

\author{
Nicholas Scott And Tetsu Hara \\ Graduate School of Oceanography, University of Rhode Island, Narragansett, Rhode Island \\ EDWARD J. WALSH \\ Observational Science Branch, NASA Goddard Laboratory for Hydrospheric Processes, Wallops Island, Virginia \\ Paul A. Hwang \\ Oceanography Division, Naval Research Laboratory, Stennis Space Center, Mississippi
}

(Manuscript submitted 5 January 2004, in final form 30 July 2004)

\begin{abstract}
A new wavelet analysis methodology is proposed to estimate the statistics of steep waves. The method is applied to open ocean wave height data from the Southern Ocean Waves Experiment (1992) and from a field experiment conducted at Duck, North Carolina (1997). Results show that high wave slope crests appear over a wide range of wavenumbers, with a large amount being much shorter than the dominant wave. At low wave slope thresholds, all wave fields have roughly the same amount of wave crests regardless of wind forcing. The steep wave statistic decays exponentially with the square of the wave slope threshold, with a decay rate that is larger for the low wind cases than the high wind cases. Comparison of the steep wave statistic with independent measurements of the breaking wave statistic suggests a breaking wave slope threshold of about 0.12 . The steep wave statistic does not scale with the cube of the wind speed, suggesting that other factors besides the wind speed also affect its level. Comparison of the steep wave statistic to the saturation spectrum reveals a reasonable correlation at moderate wave slope thresholds.
\end{abstract}

\section{Introduction}

Breaking waves are a ubiquitous phenomenon of the world's oceans. They disrupt the aqueous boundary layer causing surface renewal, thereby enhancing the diffusion of gases and heat across the air-sea interface. Breaking waves are also responsible for the dissipation of wave energy and thus directly affect the evolution of the wind wave spectrum. With advances in technology, new direct observations of the two-dimensional spatial surface wave topography have been made. These data allow for the opportunity to go beyond linear analysis and study the nonlinearity of the surface wave field, in particular, the statistics of steep and breaking waves.

Recent field studies of breaking waves have been varied. Ding and Farmer (1994) obtained breakingsurface wave data from a hydrophone array correlating breaking wave events with acoustical noise produced by bubbles in the water column. They observed a distribu-

Corresponding author address: Dr. Nicholas Scott, Applied Ocean Physics and Engineering, Woods Hole Oceanographic Institution, Mail Stop 12, 266 Woods Hole Road, Woods Hole, MA 02543.

E-mail: nscott@whoi.edu tion of the speed of breaking wave propagation and found that the results were $45 \%-75 \%$ smaller than the phase speed of the dominant wind waves. This implied that most breaking events occur with waves shorter than the dominant waves. Gemmrich and Farmer (1999) performed conductivity measurements at high sea state in the open ocean. They again found breaking wave events over a wide range of wave scales with breaking occurring predominantly at wave scales between $5 \%$ and $80 \%$ of the dominant wave scale. In addition, they found that the fraction of breaking wave events relative to the total number of dominant waves did not scale with wind speed or wave age. A scaling based on wind energy input to waves was proposed and found to collapse the diverse datasets obtained by the authors.

Banner et al. (2000) analyzed three different datasets with different wind conditions in an effort to understand the major environmental parameters that control the breaking of waves. They surmised that the probability of dominant wave breaking is strongly correlated with significant wave steepness. Banner et al. (2002) further extended the above analysis of the breaking probability to high wavenumbers with the inclusion of scales smaller than the peak wavenumber. They found 
that a spectral measure of wave steepness in the form of the spectral saturation was strongly correlated with the probability of breaking.

Phillips et al. (2001) obtained radar measurements of breaking waves off the coast of Hawaii. They found that at a set wind speed the number of events detected per unit area per unit time was of the same order as that found by Ding and Farmer (1994). However, the distribution of scales of breaking wave events was narrower with the fastest breaking wave events possessing a speed of about $60 \%$ of the dominant wave speed. In addition, they calculated $\Lambda$ (c)dc, the average length of breaking wave crests per unit surface area of ocean surface traveling at velocities in the range $(\mathbf{c}, \mathbf{c}+\mathbf{d c})$. They found that the amount of breaking waves increased as the scale decreased, indicating the importance of small-scale breaking to energy dissipation.

Melville and Matusov (2002) obtained images of ocean whitecaps from an aircraft. They tracked the evolution of whitecaps using image velocimetry and thus were able to calculate the breaking wave statistic, $\Lambda(\mathbf{c})$. Their observations indicated an exponential dependence of $\Lambda(\mathbf{c})$ on $\mathbf{c}$ with a local approximation consistent with $\Lambda(\mathbf{c}) \propto \mathbf{c}^{-6}$ for large values of $\mathbf{c}$ and $\Lambda(\mathbf{c}) \propto \mathbf{c}^{-1}$ for small values of $\mathbf{c}$.

While most of the previous observational studies used whitecaps or bubbles to detect breaking wave events, new mathematical methods of data analysis have been exploited in trying to detect breaking wave phenomena from the wave height record. In particular, Liu (1994) used the wavelet transform to analyze ocean wave data taken with a wave wire mounted on a buoy. He proposed to designate as a breaking wave any wave that possessed a value for $a \omega^{2}$ that exceeds a limiting fraction of the gravitational acceleration, where $a$ is the wave amplitude and $\omega$ is the angular frequency obtained from averaging over a selected high-frequency region of the wavelet transform.

Although Liu's (1994) approach is limited to a narrowband wave system and cannot be applied to open ocean surface wave data, the wavelet transform approach, in principle, should be suitable for detecting wave breaking. This is because previous theoretical studies (Dold and Peregrine 1986; Banner and Tian 1998) suggest that breaking wave events are associated with wave groups with strong nonlinearity rather than with a single steep wave, and wave groups can be detected by the wavelet transform.

Dold and Peregrine (1986) examined the evolution of wave groups numerically using a fully nonlinear twodimensional, free-surface computational model. They found that whether the initial wave group evolved to breaking was dependent not only on the initial carrier wave slope but also on the number of waves $N$ in the modulation interval. For a given $N$, breaking always occurred above a particular wave slope threshold value. Banner and Tian (1998) performed a numerical study of the onset of wave breaking for unforced nonlinear modulated wave groups through the use of a wave slope threshold variable. Using the same code as Dold and Peregrine (1986), they examined the evolution of wave groups in terms of the relative wave growth rates of the local mean energy and momentum densities. They found evidence of a universal threshold for the local relative growth rates of the mean momentum and energy densities that differentiates between the breaking and nonbreaking wave groups.

In this study, we propose a new approach to estimate the statistics of steep wave events (wave groups of large amplitude) by applying the wavelet transform to broadband open ocean wave fields. We make use of the spatial wave topographic data obtained during the Southern Ocean Waves Experiment (SOWEX) and the experiment conducted off Duck, North Carolina, in September 1997 (hereafter termed the DNC experiment). The results are then used to examine how the statistic of nonlinear wave groups may correlate with the true breaking wave statistic. In this study, we present a one-dimensional data analysis based on the assumption that all steep wave events propagate in the mean wind direction. In a companion paper (Scott et al. 2005), we examine the directionality of the steep wave statistics.

\section{Experiments}

The SOWEX was an international collaboration that focused on the acquisition of meteorological data over the Southern Ocean for a range of wind speeds. The objective of the experiment was to increase the present knowledge of the variability of the wind stress under a variety of sea states and wind speeds. An aircraft with a Scanning Radar Altimeter flew over the Southern Ocean off the southwest coast of Tasmania, Australia. The Scanning Radar Altimeter is a device that gathers two-dimensional information of the sea surface by scattering high-frequency waves off it. The ocean fetch was unlimited and the 10-m mean wind speed decreased from almost 26 to $2 \mathrm{~m} \mathrm{~s}^{-1}$ over the expanse of the experiment. Details about the instrument and experiment can be found in Banner et al. (1999).

The DNC experiment was a field experiment conducted near Duck, North Carolina, in September 1997. During this experiment, an airborne scanning laser ranging system (ATM) captured the spatial topography of ocean surface waves. The dataset consists of twodimensional images of surface wave height with the wind blowing $45^{\circ}$ with respect to the line of flight. The wind was quasi steady with a speed of $9.5 \mathrm{~m} \mathrm{~s}^{-1}$. Details concerning the experiment can be found in Hwang et al. (2000).

\section{Data analysis}

\section{a. Definition of the steep wave statistic}

The steep wave statistic in this study is defined based on the formulation of the breaking wave statistic by 
Phillips (1985). Phillips (1985) proposed a distribution function $\Lambda$ (c) such that $\Lambda$ (c)dc represents the average total length per unit surface area of breaking wave fronts that have intrinsic velocities in the range $\mathbf{c}$ to $\mathbf{c}+$ dc. Alternatively, a distribution function may be defined in terms of the wavenumber such that $\Lambda(\mathbf{k}) \mathbf{d k}=$ $\Lambda(k, \theta) k d k d \theta$ is the average total length per unit surface area of breaking wave fronts that have wavenumbers in the range $\mathbf{k}$ to $\mathbf{k}+\mathbf{d k}$. Here the wavenumber $\mathbf{k}$ is defined as the spatial change in wave phase with distance. In this study, the steep wave statistic, $\Lambda_{T}(k, \theta)\left(\right.$ or $\Lambda_{T}(k)$ $\left.=\int \Lambda_{T}(k, \theta) k d \theta\right)$, is defined as the total length of steep wave fronts with wave slope exceeding a set threshold $T$ per unit surface area per unit wavenumber vector (or per unit wavenumber). The steep wave statistic, $\Lambda_{T}(k)$ is a dimensionless quantity that, when integrated over all wavenumbers yields $\Gamma=\int \Lambda_{T}(k) d k$, the total length of steep wave fronts above wave slope threshold $T$ per unit surface area.

In general, the slope of an individual wave cannot be determined uniquely for random seas with a broadbanded spectrum. Thus, the wave slope is defined using the wavelet transform such that the estimated slope of an individual steep wave event is, in fact, the average wave slope of a small group of waves that are detected by the wavelet transform.

\section{b. Wavelet transform}

The original motivation for the use of the wavelet transform was the desire to obtain wavenumber information from a signal $f(u)$, where $u$ is a space variable, without excessive loss of resolution in space. This desire was not fulfilled by the Fourier transform, which gives information about the wavenumber content of a signal but gives no information about the location of events at specific wavenumbers in space. The wavelet transform of signal $f(u)$ in this study is defined as

$W f(a, s)=\operatorname{Re}\left[\int_{-\infty}^{\infty} f(u) \frac{1}{a^{2}} \Psi\left(\frac{u-s}{a}\right) d u\right], a>0$,

where

$$
\Psi(a, s)=\Psi(s / a)=e^{-i K_{0} s / a} e^{-1 / 2(s / a)^{2}}, K_{0}=5
$$

is the Morlet wavelet, $a$ is the scale, and $s$ is the location. The Morlet wavelet is an analytic solution of the linear wave theory equations whose characteristic feature of resembling a wave group warrants its use in understanding the local features in wave data. The wavelet transform possesses two aspects. When applied to a signal it effectively searches the signal and finds regions where the data looks like itself; that is, it searches for local regions of the signal that have wavelike characteristics. The normalization used is not the classical $a^{-1 / 2}$ normalization that preserves energy but rather a normalization that preserves wave slope. For example, if the signal is interpreted as wave height, the local peak value of the inner product of the signal and the Morlet wavelet produces a measure of the average wave slope over the support of the wavelet. Thus, a signal with a high wave slope event with its scale $a_{0}$ and its crest position $s_{0}$ will have a wavelet transform characterized by a large peak value of $W f$ at $\left(a_{0}, s_{0}\right)$, and the peak value is proportional to the average slope of the signal in the neighborhood of $s_{0}$. Furthermore, two sinusoidal signals whose wave slopes, $S=A k$ (where $A$ is wave amplitude and $k$ is wavenumber), are equal will have equal peak values of $W f(a, s)$ at each of the signals' respective scales (see the appendix). The preservation of the signal's average wave slope in the "local" sense as described above allows for the detection of high wave slope crests that are part of wave groups in the data.

The wavelet analysis applied here is very different from other conventional analyses, which attempt to quantify the local characteristics of waves by the examination of individual waves identified by the crossing of the zero level. Though the zero-crossing methodology is one of the traditional methods of calculating the probability of breaking, the issue of how to deal with small-scale riding waves exists. In addition, it allows for only one breaking wave event per wave cycle. The wavelet analysis methodology outlined here, on the other hand, is a multiscale matched filtration process and allows for many steep wave events over the dominant wave cycle.

\section{c. Estimation of the steep wave statistic, $\Lambda_{\mathrm{T}}(\mathrm{k})$}

\section{1) WAVELET ANALYSIS OF SIGNAL}

The SOWEX dataset is divided into four individual subsets designated by the experimental day. Each of these subsets in turn contains wave height images with different resolutions in the along-flight and cross-flight directions. Initially, the SOWEX and the DNC datasets were preprocessed. A nine-point box filter was applied to the DNC dataset for the purpose of smoothing out the high frequency noise. The occasional dropouts, which were never more than three sample points long, in the SOWEX dataset were linearly interpolated over. The SOWEX and DNC datasets were rotated and then bilinearly interpolated such that the $y$ axis is in the direction of the mean wind and all datasets possess the same resolution in the along-wind and cross-wind directions.

For each dataset analyzed, columns of the data, which constitute data in the direction of the wind, were stripped off. These data vectors subsequently underwent the wavelet transform. Wavelet analysis was performed over a set of discrete scales (with a constant $\Delta a$ ) that extend from a scale larger than the dominant wavelength down to a scale before the Fourier spectral noise floor in the data appears. For the SOWEX and DNC datasets the largest scales corresponded to 1.4 and 1.8 times the dominant wavelength, respectively. The cut- 
off scale was obtained from the inspection of the Fourier power spectrum. For the DNC dataset the wavelet transform at scale values with equivalent wavenumbers above $0.9 \mathrm{rad} \mathrm{m}^{-1}$ were not taken. For the SOWEX dataset the high wavenumber cutoff was $0.09 \mathrm{rad} \mathrm{m}^{-1}$.

Each column vector of the wave height image admits a two-dimensional function, $W f(a, s)$, dubbed the wavelet transform. The conversion of the scale $a$ used in the wavelet analysis to the real wavenumber scale $k$ is obtained via a conversion constant $C$. The wavenumber associated with the wavelet at scale $a$ is taken to be the wavenumber associated with the peak in the power spectrum of the wavelet. The one-to-one relationship between wavenumber and wavelet scales can be expressed as

$$
k=\frac{C}{a} .
$$

The Morlet wavelet at different scales and their respective Fourier power spectra are shown in Fig. 1.

A typical example of a wavelet transform is shown in Fig. 2. A contour plot of the wavelet transform along with a plot of the analyzed signal is shown from 10 June of SOWEX. The wavelet transform $W f(a, s)$ contains an array of high wave slope events associated with wave groups. To obtain a distribution of these events, a wave slope threshold is applied over it. All points with a wavelet transform value above a set threshold are selected. These points appear as aggregates in local regions of $W f(a, s)$, enclosed by the dashed lines. From these groups of points, the point of highest value is sought. The highest points or local maxima throughout the wavelet transform are obtained by using a ninepoint box filter. The filter is moved throughout the transform and from these groups selects only those points that are larger than the surrounding eight points. The local maxima that satisfy the condition of being above the set threshold appear in the figure as asterisks. These asterisks are defined as individual steep wave events in the subsequent analyses. It is noteworthy that more than one local maximum (asterisk) can exist within a single region enclosed by a dashed line when the threshold is set low.

The wave slope threshold is understood in terms of the wave slope associated with individual sinusoids. Thus the wave slope of an event associated with a wave group calculated via the wavelet transform is an equivalent wave slope or the wave slope of a sinusoid with an average wavenumber and average amplitude equivalent to the Morlet wavelet. The conversion from the wavelet transform threshold to the equivalent wave slope threshold is accomplished by first taking a pure sinusoid $f(u)=A \sin k u$, of known wave amplitude $A$ and wavenumber $k$ and obtaining the wavelet transform of the signal, $W f(a, s)$. The peak value of the wavelet transform is then related to the wave slope $A k$ to determine the conversion constant $\gamma$ :

$$
\gamma=\frac{A k}{\max [W f(a, s)]} .
$$
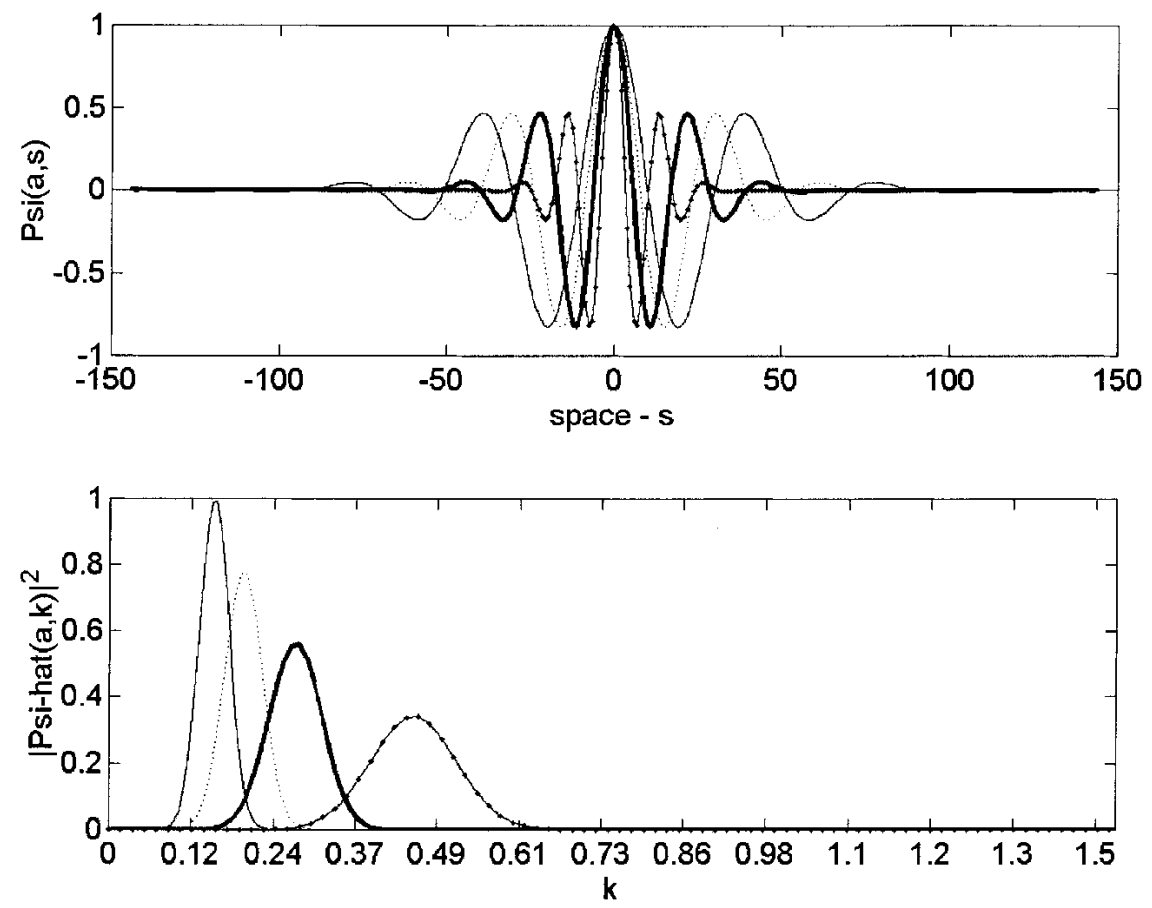

FIG. 1. (top) Morlet wavelets at different scales and (bottom) their respective Fourier power spectra. Scales $a=28$ (thin line), 21 (dotted line), 14 (thick line), and 7 (thin line with dots). 


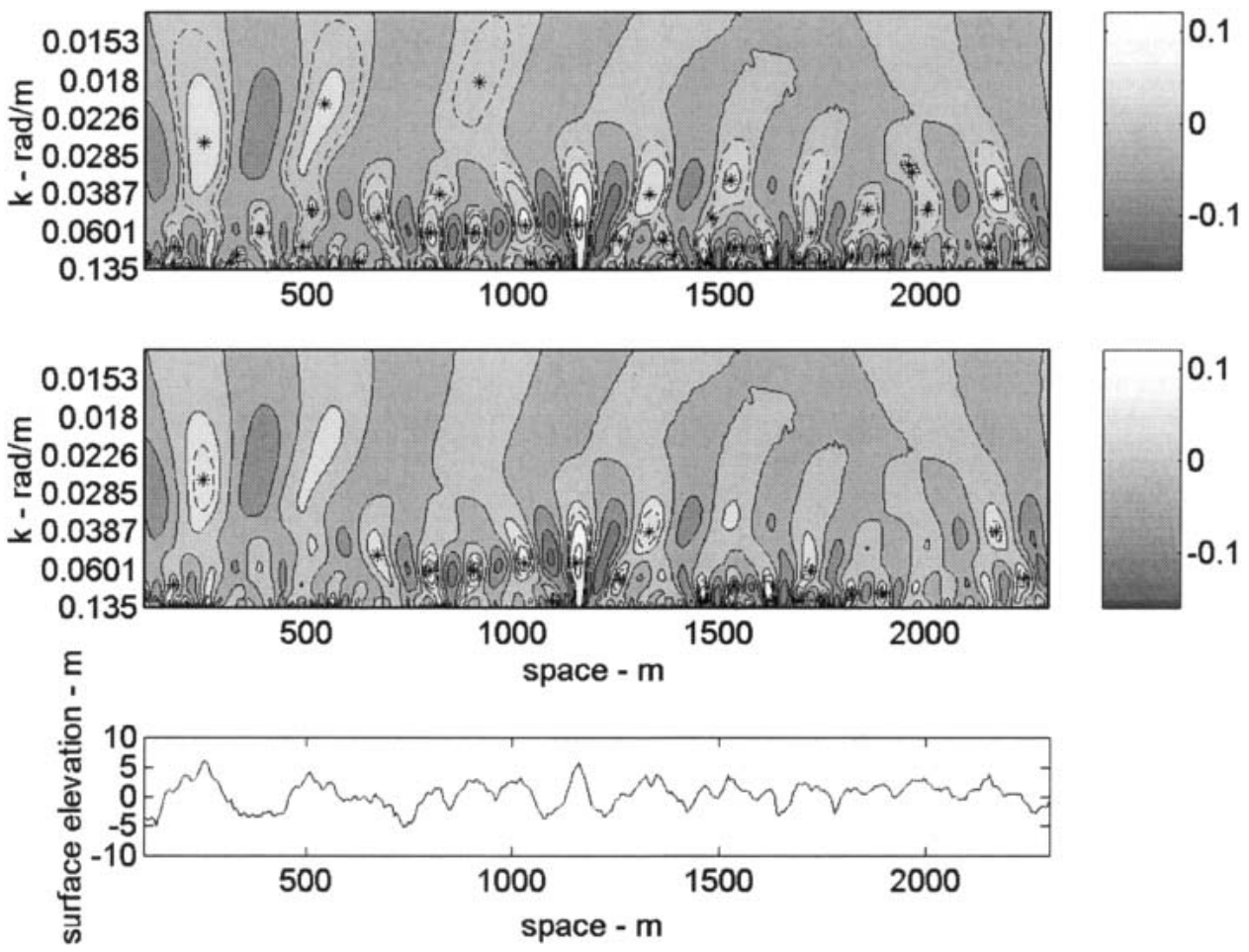

Fig. 2. (bottom) The 10 Jun SOWEX surface elevation signal and (top), (middle) contour plots of its wavelet transform. Solid contour lines are drawn at every 0.04. Dashed contour lines are at wave slope threshold of (top) 0.02 and (middle) 0.06 . Wave crests exceeding wave slope threshold of (top) 0.02 and (middle) 0.06 are marked with asterisks.

With real surface wave height data, this constant is used to convert from the local maximum value of the wavelet transform to the real equivalent local wave slope threshold $T$.

Each high wave slope event in a data vector, denoted by asterisks in Fig. 2, has associated with it a scale value and an along-wind position value. The scale values together form $D_{T}(a)$, where $D_{T}$ is the number of events of scale $a$ exceeding a set wave slope threshold $T$. The same method of analysis outlined above is applied to all of the data vectors (with index $n$ ) that are part of the full two-dimensional wave height image to obtain a set of distributions $D_{T}^{n}(a)$.

\section{2) Formation of THE STEEP WAVE STATISTIC}

The lambda function $\Lambda_{T}(a)$ at a set wave slope threshold $T$ as a function of scale $a$ is calculated first by summing the distributions over $n$ and then normalizing the result.

Thus, the final result for $\Lambda_{T}(a)$ is

$$
\Lambda_{T}(a)=\frac{\sum_{n=1}^{N} D_{T}^{n}(a) \times \Delta x}{\tilde{A} \times \Delta a} .
$$

Here, the cross-wind sampling distance $\Delta x$ is taken to be the length of the high wave slope crest associated with the steep wave event, $\Delta a$ is the differential scale, $N$ is the total number of data vectors, and $\tilde{A}$ is the total area of the image analyzed.

For a single image, $\Lambda_{T}(k)$ is calculated from $\Lambda_{T}(a)$ by applying a normalization factor. Since the differentials, $d a$ and $d k$, are opposite in sign, $\Lambda_{T}(k)$ and $\Lambda_{T}(a)$ are related to each other by the equation:

$$
\Lambda_{T}(k) d k=-\Lambda_{T}(a) d a .
$$

Therefore, $\Lambda_{T}(k)$ can be written as

$$
\Lambda_{T}(k)=-\Lambda_{T}(a) \frac{d a}{d k},
$$

where $a$ is related to $k$ via Eq. (3). Thus $\Lambda_{T}(k)$ can be written as

$$
\Lambda_{T}(k)=\Lambda_{T}(a) \frac{C}{k^{2}}=\Lambda_{T}(a) \frac{a^{2}}{C} .
$$

The result $\Lambda_{T}(k)$, the steep wave statistic, is a dimensionless function. This function is dependent on the wave slope threshold $T$ applied and is quantitatively a measure of the amount of high wave slope events in the wave field. The main assumption implicit in this calculation is that all wave fronts are perpendicular to the mean wind. (We will demonstrate the validity of this 
assumption in the companion paper.) This quantity is averaged over many datasets to obtain the averaged steep wave statistic. For the DNC dataset, 23 realizations of the steep wave statistic were averaged. For the SOWEX data subsets, hereafter identified as the 10,12, 13 , and 14 June experiments, 15, 18, 36, and 6 realizations, respectively, were averaged.

\section{3) DOPPLER CORRECTION FOR THE STEEP WAVE STATISTIC}

The Doppler shift of the waves due to the aircraft motion is a major source of error. The error associated with this effect is estimated in terms of the apparent and actual value for $\Lambda_{T}(k)$. From the steep wave statistic associated with the DNC and SOWEX datasets the wavenumbers at a set threshold are selected along with their corresponding values for $\Lambda_{T}(k)$. The Doppler shift effect in the value of $\Lambda_{T}(k)$ is traced as the wavenumber changes due to the Doppler effect. The steep wave statistic can be written as

$$
\Lambda_{T}(k)=\frac{L}{\tilde{A} \times \Delta k},
$$

where $L$ is the total length of steep wave crests at a specific wave slope threshold $T$ over a small interval $\Delta k$ in wavenumber. Depending on the aircraft speed and direction, the apparent wavenumber $k_{\text {app }}$ is shifted from the true wavenumber $k_{\text {tru }}$ along with an accompanied interval change from $\Delta k_{\text {tru }}$ to $\Delta k_{\mathrm{app}}$. In addition, the shift in wavenumber produces a shift in the wave slope threshold $T_{\text {tru }}$ to a new apparent wave slope threshold $T_{\text {app }}$ since the surface elevation is not affected by the Doppler shift. All three corrections were applied to obtain the true values of $\Lambda_{T}(k)$. This Doppler correction was also performed assuming that all wave fronts are perpendicular to the mean wind.

\section{Results and discussion}

\section{a. Detection of steep wave crests}

The wavelet analysis algorithm of the surface wave topography data vectors is successful at pinpointing high wave slope crests that are part of steep wave groups. Figure 2 shows a space series taken from 10 June of SOWEX along with a contour plot of the wavelet transform values. Asterisks identify high wave slope events that exceed a wave slope threshold of 0.02 (Fig. 2, top) and 0.06 (Fig. 2, middle). The algorithm is able to perform a multiscale filtration of the data to reveal steep wave crests at both large and small scales. The steep wave slope events identified by the asterisks at small and large wavenumbers are clearly correlated with large and small steep wave crests that are part of wave groups in the surface height signal. Some asterisks do not lie directly at the points of maximum surface elevation in the signal. This is due to the inner product associated with the convolution. It attempts to make the best fit of the Morlet wavelet to the data and occasionally falls short of locating wave crests in agreement with the actual crest locations.

\section{b. Characteristics of the steep wave statistic}

The estimates of the steep wave statistic $\Lambda_{T}(k)$ for the four SOWEX cases and the DNC experiment are shown without the Doppler correction in Fig. 3 and with the Doppler correction in Fig. 4. Although the results are qualitatively very similar, the estimated values of $\Lambda_{T}(k)$ may vary by a factor of 2 or more due to the Doppler correction. We therefore make use of the results with the Doppler correction in the subsequent analyses.

The curves in Fig. 4 in general display low values of $\Lambda_{c T}(k)$ below the dominant wavenumber (indicated by the vertical lines) and increased amounts of steep wave crests at high wavenumbers. The results of the steep wave statistic bear qualitative resemblance to the breaking wave results of Ding and Farmer (1994). They found the average scale of breaking to be much smaller than the dominant wind wave. The steep wave statistic is consistent with this result with the peak in the steep wave statistic occurring at a much higher wavenumber than the dominant wind wave.

What is clearly shown in these plots is that all datasets have approximately the same average length of wave crests per unit area per unit wavenumber, regardless of the wind forcing or the wave field, at the lowest wave slope threshold of 0.01 . Thus, statistically the total number of wave crests is similar for all cases if you count all wave crests (low and high wave slope waves) in the wave field.

Differences in $\Lambda_{c T}(k)$ become apparent with increases in the wave slope threshold $T$. For the SOWEX dataset, Fig. 4 shows that $\Lambda_{c T}(k)$ at various wavenumbers decreases much more rapidly with increasing $T$ for the low wind cases than for the high wind cases. This is to be expected since, to the first order, the higher wind cases tend to possess larger amounts of high wave slope events.

Figure 5 shows that $\Lambda_{c T}(k)$ at a fixed $k$ from both the SOWEX and DNC datasets decays exponentially with wave slope threshold squared, that is, it approximately follows the form of

$$
\Lambda_{c T}=\Lambda_{o} e^{-p T^{2}},
$$

with a fixed exponent $p$ and multiplicative constant of $\Lambda_{o}$. Here, we have chosen $\Lambda_{c T}\left(k=0.04 \mathrm{rad} \mathrm{m}^{-1}\right)$ from the data of the SOWEX and $\Lambda_{c T}\left(k=0.4 \mathrm{rad} \mathrm{m}^{-1}\right)$ from the DNC data since these wavenumbers are significantly beyond the dominant wavenumber but above the point of noise for all the datasets. The value of the exponent $p$ can be determined by fitting the data to a straight line in the least squares sense, and is designated as $p_{\text {fit }}$. The estimated values of $p_{\text {fit }}$ for each day of 


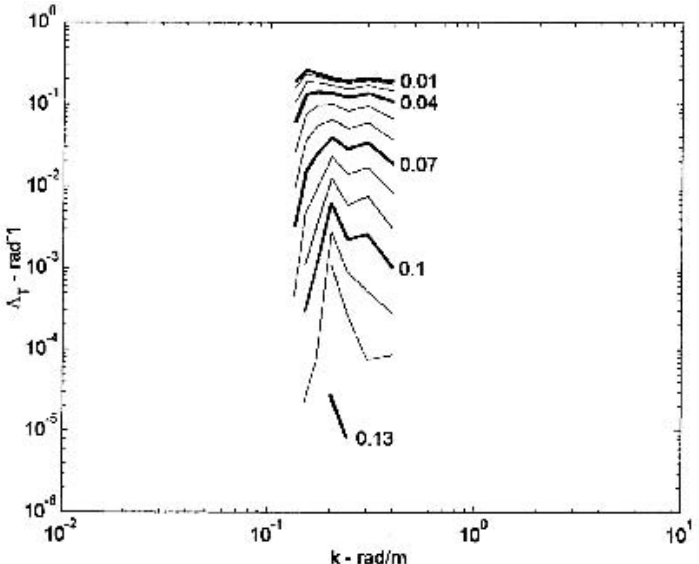

a)

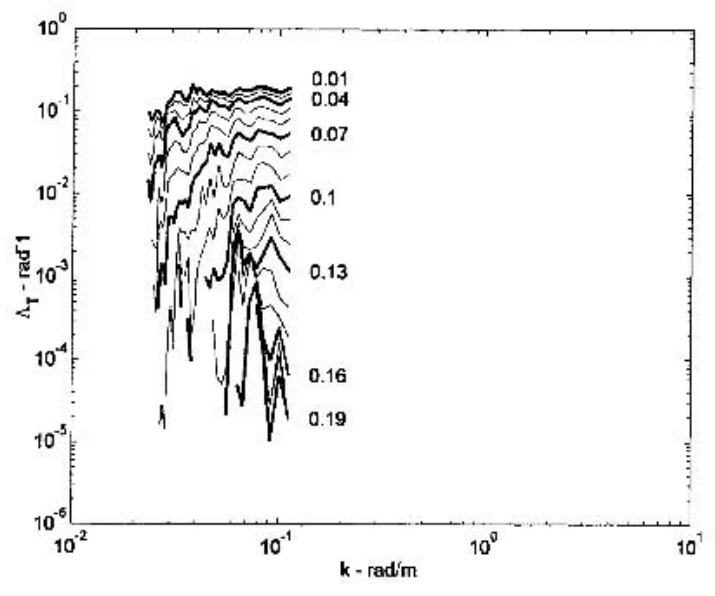

c)

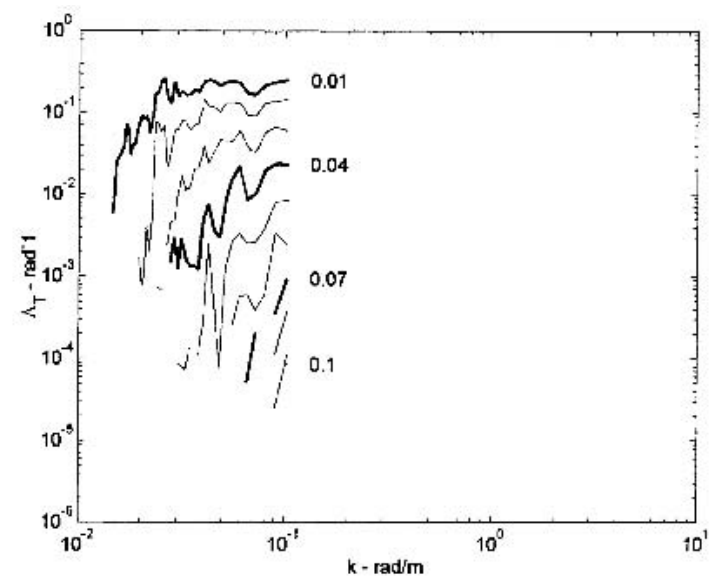

e)

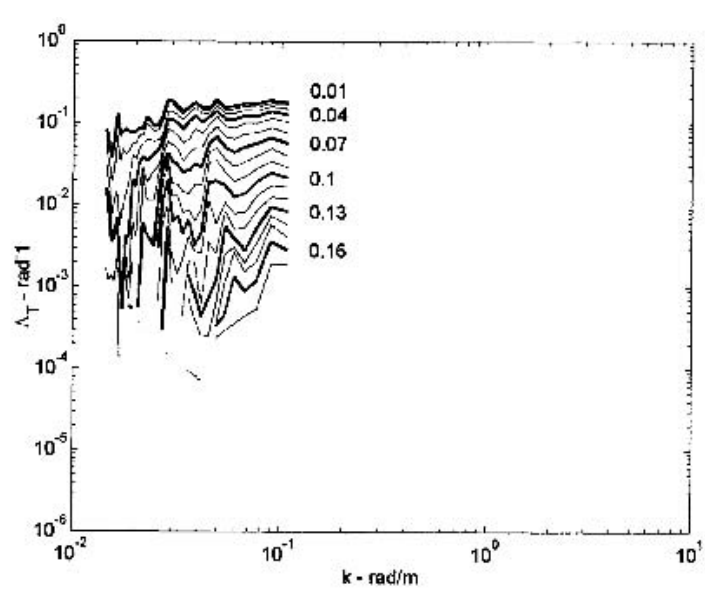

b)

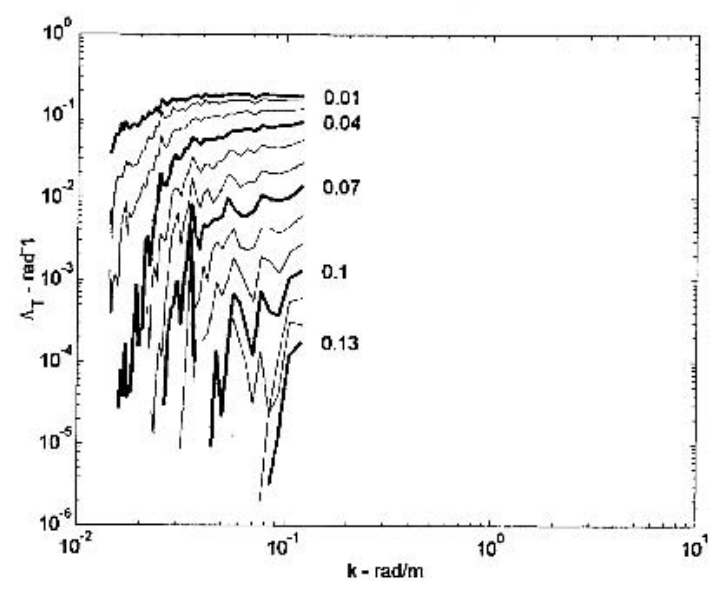

FIG. 3. Steep wave statistic $\Lambda_{T}(k)$ vs $k$ for different wave slope threshold values. Numbers in the figure indicate slope threshold T. (a) DNC experiment (mean wind speed $9.5 \mathrm{~m} \mathrm{~s}^{-1}$ ), (b) $10 \mathrm{Jun}$ SOWEX (mean wind speed $25 \mathrm{~m} \mathrm{~s}^{-1}$ ), (c) 12 Jun SOWEX (mean wind speed $24 \mathrm{~m} \mathrm{~s}^{-1}$ ), (d) 13 Jun SOWEX (mean wind speed 8.5 $\mathrm{m} \mathrm{s}^{-1}$ ), and (e) 14 Jun SOWEX (mean wind speed $6 \mathrm{~m} \mathrm{~s}^{-1}$ ). 


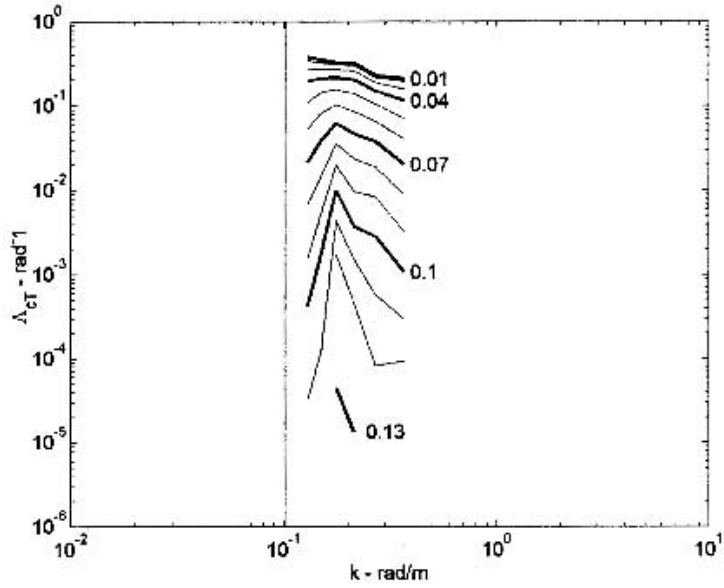

a)

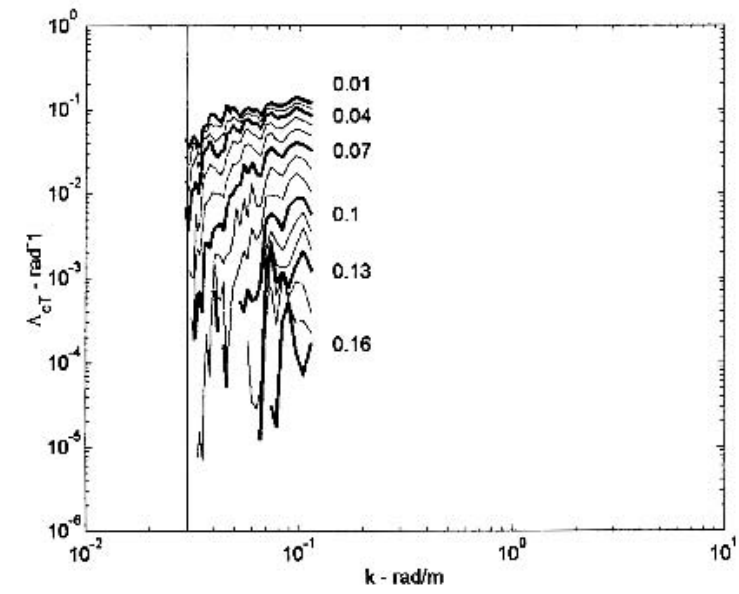

c)

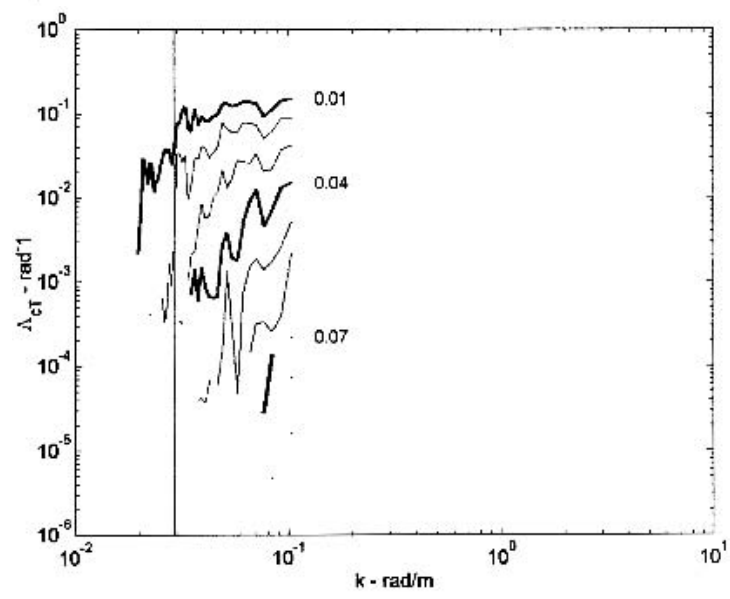

e)

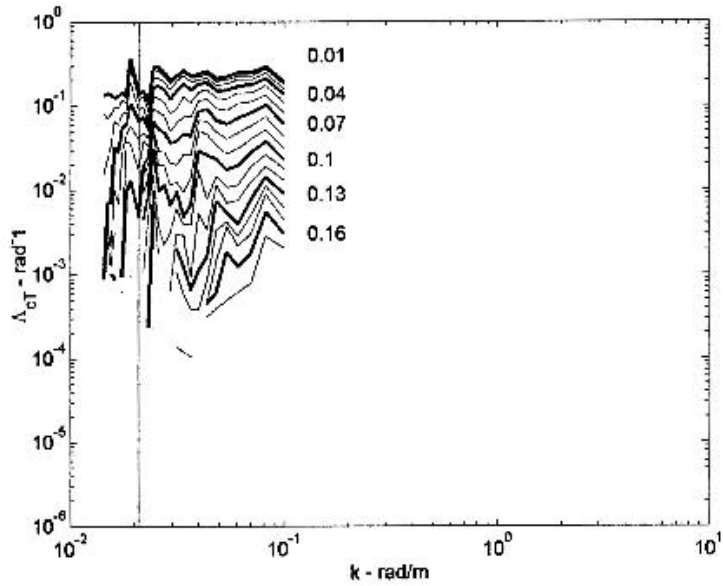

b)

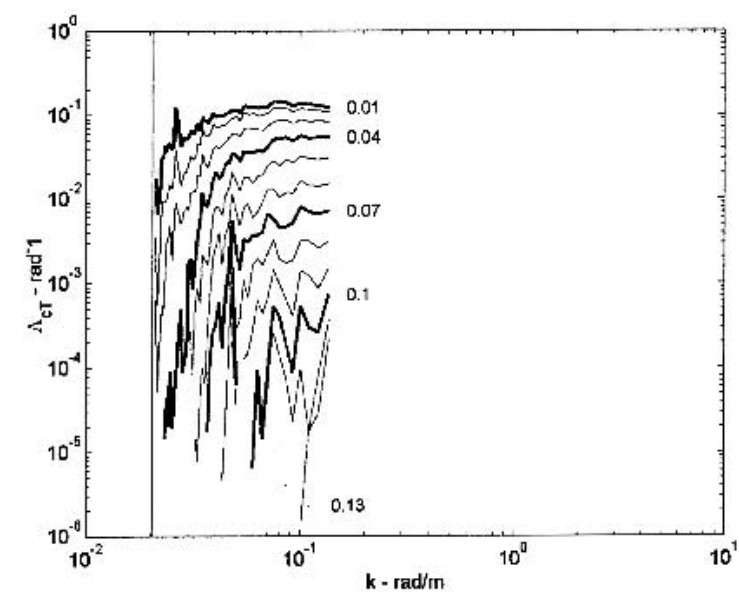

d)

FIG. 4. As in Fig. 3 but for corrected steep wave statistic $\Lambda_{c T}$ vs $k$. Vertical line indicates dominant wavenumber. 


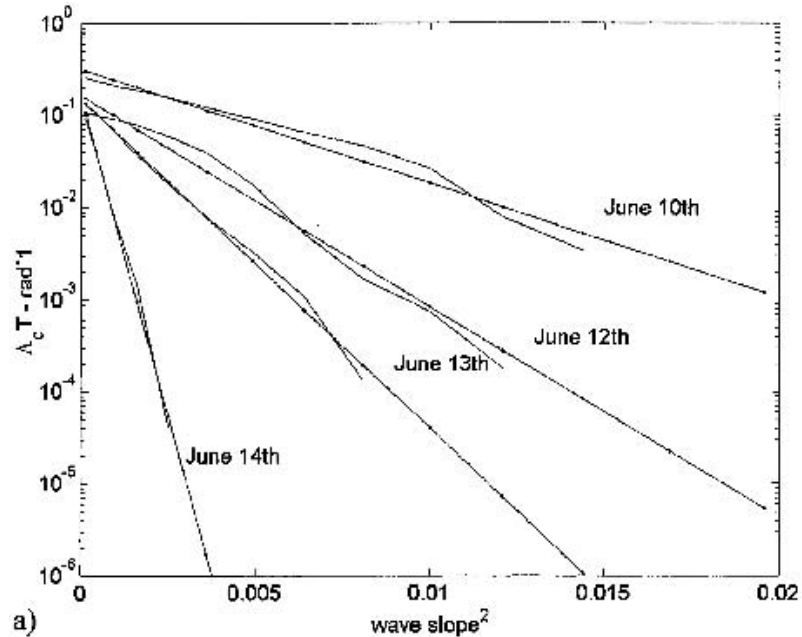

a)

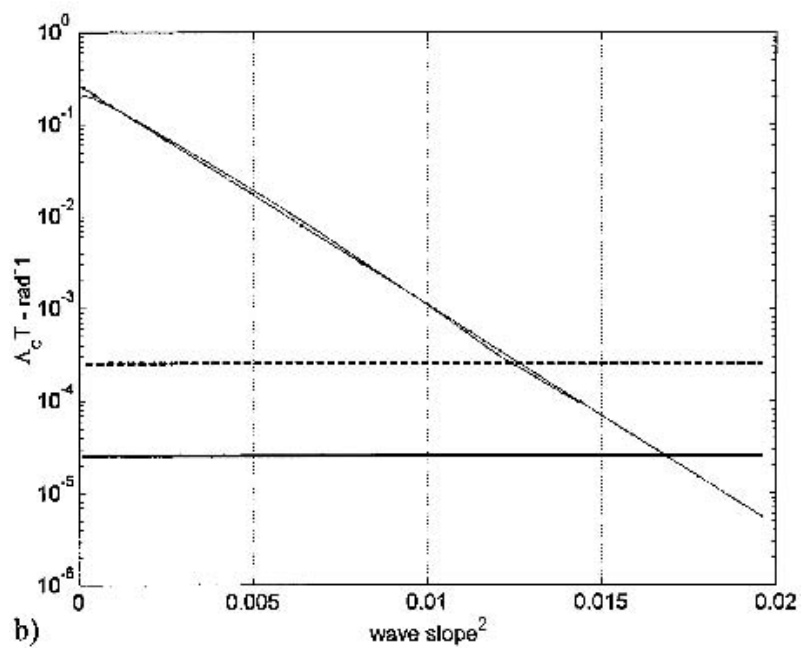

FIG. 5. The steep wave statistic $\Lambda_{c T}(k)$ vs wave slope squared $\left(T^{2}\right)$. Data designated by solid lines. Least squares fit designated by dots connected by solid lines. (a) $\operatorname{SOWEX}\left(k=0.04 \mathrm{rad} \mathrm{m}^{-1}\right)$ and (b) DNC experiment $\left(k=0.4 \mathrm{rad} \mathrm{m}^{-1}\right)$. The thick solid line is Phillips et al.'s (2001) estimate of $\Lambda$. The thick dashed line is Melville and Matusov's (2002) estimate of $\Lambda$.

SOWEX are plotted against the friction velocity in Fig. $6 \mathrm{a}$. The general decreasing trend in the exponent $p_{\text {fit }}$ with increasing $u_{*}$ is observed as expected. However, $p_{\text {fit }}$ does not seem to be a simple function of $u_{*}$; the data at the two lowest wind days show very different results of $p_{\text {fit }}$ even if the wind friction velocity is almost the same.

\section{c. Comparison of the steep wave statistic with the theory by Cartwright and Longuet-Higgins}

Cartwright and Longuet-Higgins (1956) considered the problem of the expected number of maxima and minima in a time series of the sea surface displacement and their probability distribution. They found that, for a narrowband random process, the probability density

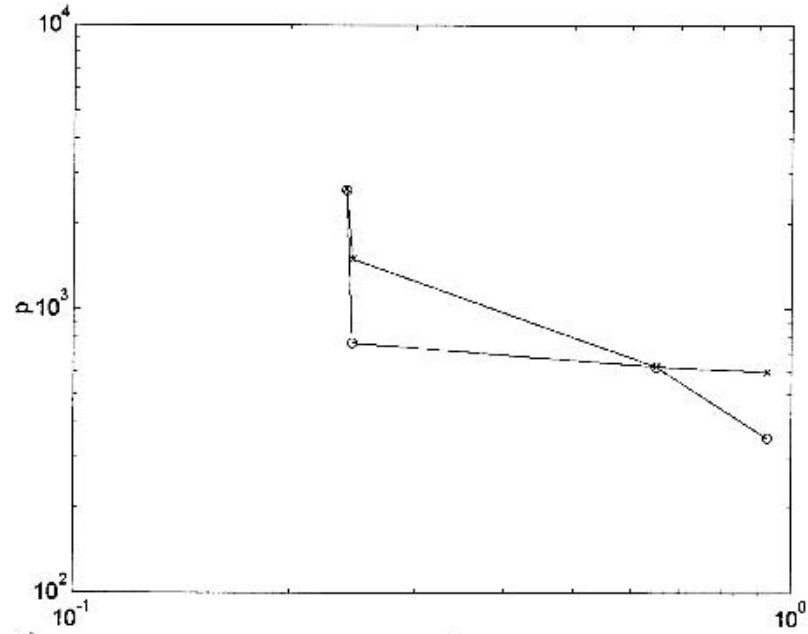

a)

$u_{4}-m / s$

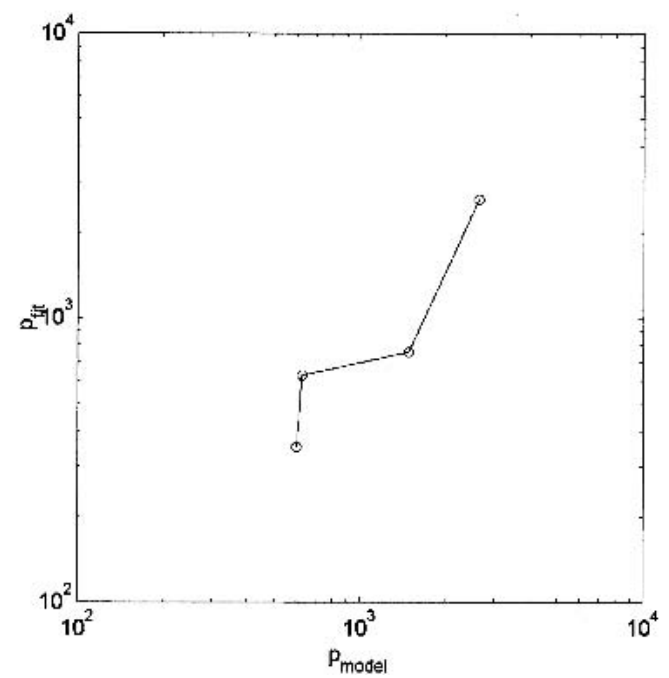

FIG. 6. (a) Exponents $p_{\text {fit }}$ (circles) obtained from the least squares fit of $\Lambda_{c T}(k)$ vs $T^{2}$ at $k=0.04 \mathrm{rad} \mathrm{m}^{-1}$, and $p_{\text {model }}$ (crosses) obtained from the theoretical model, plotted against $u_{*}$ for SOWEX. (b) Exponent $p_{\text {fit }}$ vs $p_{\text {model }}$ for SOWEX. Correlation coefficient $\rho=0.94$.

function for extreme surface displacements is the Rayleigh distribution

$$
f_{\max }(\xi)=\xi e^{-\xi^{2} / 2}
$$

with $\xi=\zeta_{\max } / m_{0}$, where $\zeta_{\max }$ is a variable delineating local positive maxima and $m_{0}$ is the root-mean-square surface displacement. According to their theory, the probability density function $\tilde{f}_{\max }(\tilde{\xi})$ for extreme surface displacements that exceed a threshold $\tilde{\xi}$ becomes

$$
\tilde{f}_{\max }(\tilde{\xi})=\int_{\tilde{\xi}}^{\infty} \xi e^{-\xi^{2} / 2} d \xi=e^{-\tilde{\xi}^{2} / 2} .
$$

For a narrowband system, a local displacement maximum that exceeds $\tilde{\xi}$ should be roughly equivalent to a local wave crest whose wave steepness exceeds $k_{0} m_{0} \tilde{\xi}$, 
where the representative wavenumber of the system is $k_{0}$. Therefore, the same statistic can be expressed as

$$
\tilde{f}_{\max }(T)=e^{\left[-1 / 2\left(T / k_{0} m_{0}\right)^{2}\right]}
$$

that is, it decays exponentially with the square of the wave slope threshold $T$. It is noteworthy that this predicted form is consistent with our observational results of $\Lambda_{c T}\left(k=k_{0}\right)$ shown in Fig. 5. Furthermore, our observations show that $\Lambda_{c T}\left(k=k_{0}\right)$ decays exponentially with the square of the wave slope threshold $T$ over a relatively large range of $k$ (roughly from the peak wavenumber $k_{p}$ to 5 times the peak wavenumber), although the theory of Cartwright and Longuet-Higgins (1956) should be applicable only for a narrowband spectrum in the vicinity of the peak wavenumber.

Equation (12) allows for the estimation of the constant exponent $p$ that multiplies $T^{2}$, if the representative wavenumber $k_{0}$ and the root-mean-square surface displacement $m_{0}$ are known. From the experimental data, $k_{0}$ has been chosen to be $0.04 \mathrm{rad} \mathrm{m}^{-1}$ for the SOWEX data. It is not possible to unambiguously determine $m_{0}$ from the observed broadband spectra because we may integrate the wave height wavenumber spectrum over an arbitrary range $\Delta k$ in the vicinity of $k_{0}$ to estimate $m_{0}$. Here, we have fixed $\Delta k$ empirically to 0.001 so that the exponent $p$ determined from Eq. (12), designated as $p_{\text {model }}$, best matches $p_{\text {fit }}$ determined from the observations. As long as the same $\Delta k$ value is used for all cases, we may examine the relative trend of $p_{\text {model }}$ versus $p_{\text {fit }}$. Figure $6 \mathrm{~b}$ indeed shows that $p_{\text {model }}$ and $p_{\text {fit }}$ correlate reasonably well having a correlation coefficient $\rho=$ 0.94. This suggests that the theory of Cartwright and Longuet-Higgins (1956) is consistent with our observations of the steep wave statistic.

\section{d. Relationship between the steep wave statistic and the breaking wave statistic}

If it is conjectured that the steep wave statistic at a particular high wave slope threshold is equivalent to the true breaking wave statistic, the trend of $\Lambda_{c T}(k)$ from the SOWEX dataset implies that there are more breaking waves at higher wind forcing conditions. Furthermore, if the breaking wave statistic $\Lambda(k)$ is known independent of $\Lambda_{c T}(k)$, it is possible to estimate a breaking wave slope threshold by comparing the two. Phillips et al. (2001) calculated $\Lambda(c)$, with a correction for the advection of breaking wave crests by swell, over a range of wave speeds from 2.5 to $6 \mathrm{~m} \mathrm{~s}^{-1}$ at the mean wind speed of $9.3 \mathrm{~m} \mathrm{~s}^{-1}$. Melville and Matusov (2002) reported $\Lambda(c)$ at three wind speeds of 7.2, 9.8, and $13.6 \mathrm{~m}$ $\mathrm{s}^{-1}$. Therefore, the results of Phillips et al. (2001), Melville and Matusov (2002; case of wind speed $9.8 \mathrm{~m}$ $\mathrm{s}^{-1}$ ), and the DNC dataset all have the same wavenumber range and were obtained under approximately the same wind forcing conditions of $U_{10}=9 \mathrm{~m} \mathrm{~s}^{-1}$. Figure $5 \mathrm{~b}$ shows a linear fit to the natural logarithm of $\Lambda_{c T}(k=$ $k_{0}$ ) plotted with the square of the wave slope threshold at $k_{0}=0.4 \mathrm{rad} \mathrm{m}^{-1}$ for the DNC dataset. The horizontal dashed and solid lines represent the value of the breaking wave statistic at $k_{0}=0.4 \mathrm{rad} \mathrm{m}^{-1}$ obtained by Melville and Matusov (2002) and Phillips et al. (2001). The intersection between the linear fit and the horizontal lines gives two estimates of the wave slope threshold at which the steep wave statistic becomes identical to the breaking wave statistic. The corresponding wave slope threshold is between approximately 0.11 and 0.13 , with 0.12 being the midvalue. These values are much lower than the value for the traditional wave slope threshold of 0.4 first proposed by Stokes.

Dold and Peregrine (1986) conducted numerical experiments in which two-dimensional nonlinear wave packets of different initial wave slope were allowed to propagate. They were able to obtain the result of the wave slope threshold above which wave groups developed into breaking waves versus the number of waves in a wave group. It is of interest to note that for a wave packet containing five waves, the corresponding wave slope threshold is approximately 0.11 according to Dold and Peregrine (1986). This value is close to our breaking wave slope threshold extrapolated from the data.

Assuming that the steep wave statistic at the equivalent slope of 0.12 agrees with the breaking statistic, we may estimate the breaking statistic for all the SOWEX cases. Figure 7a shows the estimates of $\Lambda_{c T}(k)$ for the DNC experiment and SOWEX at $T=0.12$. Also plotted are the Phillips et al. (2001) and Melville and Matusov (2002) estimates of the breaking wave statistic. The results of the DNC experiment are close to that of the Phillips et al. (2001) and Melville and Matusov (2002) result at $U_{10}=9.8 \mathrm{~m} \mathrm{~s}^{-1}$ as expected. The SOWEX results of 10 and 12 June are much higher because the wind speed was much higher on these two days. To remove the wind speed dependence, both $\Lambda_{c T}(k)$ and $\Lambda(k)$ are scaled with the cube of the wind speed, as suggested by Melville and Matusov (2002), in Fig. 7b. The scaling collapses the datasets of Melville and Matusov (2002) while only reducing the variability between the SOWEX data within one order of magnitude or so. For example, the steep wave statistics from 10 and 12 June are significantly different even if the wind speed was similar. This difference is likely because the wave fields were at different stages of development on these two days. All of this suggests that wind speed alone may not be the appropriate scaling for the number of steep waves at $T=0.12$.

\section{e. Relationship between the steep wave statistic and the wavenumber spectrum}

To understand the relationship between the steep wave statistic and the wavenumber spectrum, $\Lambda_{c T}(k)$ versus $B(k)$, the saturation spectrum for all experimental cases is plotted in Fig. 8 at three different wave slope threshold values. The two-sided saturation spectrum is defined as 

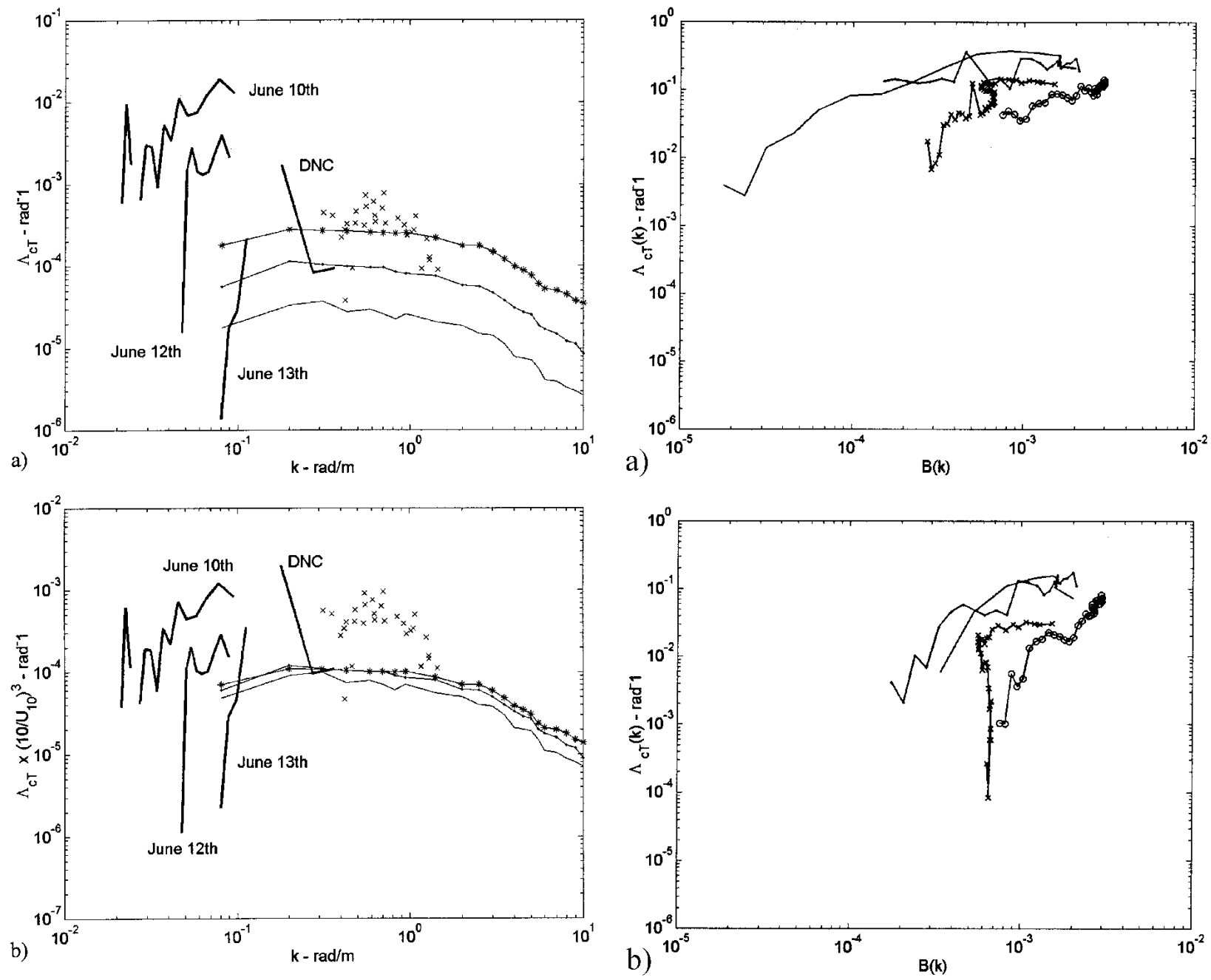

FIG. 7. (a) Estimates of $\Lambda_{c T}(k)$ at wave slope threshold $T=0.12$ for SOWEX and DNC experiment (thick lines). Phillips et al.'s (2001) estimates of $\Lambda(k)$ are designated by crosses. Melville and Matusov's (2002) estimates of $\Lambda(k)$ are shown at $U_{10}=7.2(-)$, $9.8(.-)$, and $13.6 \mathrm{~m} \mathrm{~s}^{-1}(*-)$. (b) As in (a) except $\Lambda_{c T}(k)$ and $\Lambda(k)$ are multiplied by $\left(10 / U_{10}\right)^{3}$.

$$
B(k, \theta)=k^{4} \times \tilde{S}(k, \theta),
$$

where $\tilde{S}(k, \theta)$ is the two-sided wavenumber spectrum. Doppler correction according to the methodology outlined in Banner et al. (1999) and integration over all angles yields the one-sided omnidirectional saturation spectrum, $B(k)$. Correlation coefficients were calculated for each set of curves at $T=0.01,0.05$, and 0.09 and are shown in Table 1. The correlation coefficients for each dataset increase in value with increases in the wave slope threshold from $T=0.01$ and $T=0.05$ with the data becoming sufficiently noisy at $T=0.09$ to disrupt the correlation. In particular, waves exceeding $T=0.09$ are detected only if the degree of saturation $B(k)$ is above 0.0005 . Overall, the correlation between $\Lambda_{c T}(k)$ and $B(k)$ is reasonably strong. For a given $T$ and

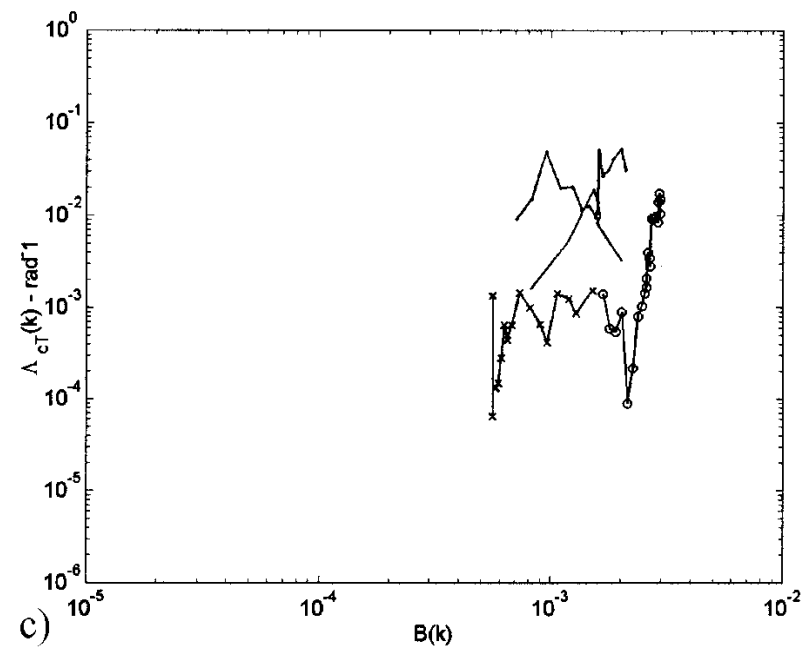

FIG. 8. $\Lambda_{c T}(k)$ vs $B(k)$ for the DNC experiment (-) and SOWEX $10(.-), 12\left(\mathrm{o}^{-}\right)$, and $13(\mathrm{x}-)$. Wave slope threshold is (a) $T=0.01$, (b) $T=0.05$, and (c) $T=0.09$. 
TABLE 1. Correlation coefficients $\rho$ for the DNC experiment and SOWEX for different wave slope thresholds.

\begin{tabular}{lccc}
\hline \hline & $T=0.01$ & $T=0.05$ & $T=0.09$ \\
\hline DNC & 0.91 & 0.92 & 0.49 \\
10 Jun SOWEX & 0.58 & 0.92 & 0.62 \\
12 Jun SOWEX & 0.91 & 0.94 & 0.73 \\
13 Jun SOWEX & 0.64 & 0.71 & 0.70 \\
\hline
\end{tabular}

$B(k)$, the value of $\Lambda_{c T}(k)$ is mostly within one order of magnitude.

Banner et al. (2002) found that the breaking probability was correlated with a nondimensional measure of the spectral steepness in the form of the saturation spectrum, $B(k)$. If the steep wave statistic at high wave slope thresholds is equivalent to the breaking wave statistic, then it is expected that $\Lambda_{c T}(k)$ at high wave slopes will be correlated with $B(k)$. Figure 8 indeed shows that $\Lambda_{c T}(k)$ increases with $B(k)$ at moderately high wave slope thresholds.

\section{Sensitivity and error analyses}

The extent to which the results presented here depend on the particular choice of wavelet in the analysis methodology is a critical issue. The use of the Morlet wavelet is justified by the need for finding an "atom" that possesses a structure similar to the actual surface wave groups. In particular, the nonlinear evolution of a wave group has been shown to be strongly related to wave breaking (Banner and Tian 1998). In keeping with this idea, this analysis is based on the search for compact wave groups in the data. The Morlet wavelet clearly has a structure that allows for the detection of wave groups in data.

A question that is extremely pertinent to the evaluation of the developed methodology is whether a finer interval of scales yields a drastically different result. We have performed data processing with twice as many scales evenly spaced over the same bounds (e.g., if $a=$ $2,4,6, \ldots$, then new scale vector $a=2,3,4,5,6, \ldots$ ) and have found very little difference in the results. Thus, it can be surmised that the increased redundancy in scale produces a negligible effect in the statistical results. The box filter used in selecting only the peak in a local neighborhood of $W f(a, s)$ yields the convergence of the two calculations.

Another issue concerning the wavelet analysis methodology is the dependence of the results on the number of waves in the Morlet wavelet packet. Morlet wavelets of wavenumbers $K_{0}=5,6,7 \ldots$ are all admissible. How robust are the results if this parameter is changed? Figure 9 shows $\Lambda_{T}(k)$ calculated through the use of the Morlet wavelet corresponding to $K_{0}=5,7$, and 9. The mother wavelet has gained more oscillations with increases in wavenumber $K_{0}$ and the width of the wavelet has expanded. This expansion allows for greater reso-
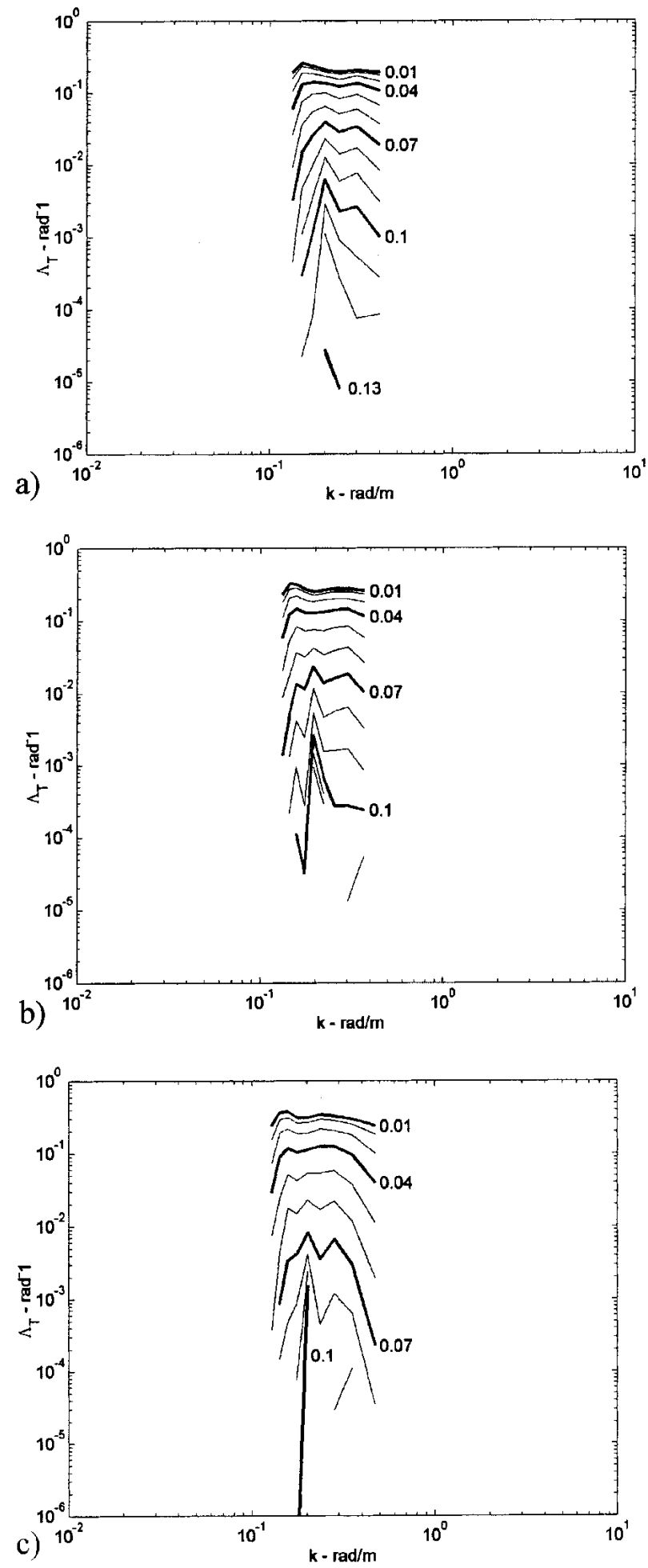

FIG. 9. The steep wave statistic $\Lambda_{T}(k)$ vs $k$ for different wave slope threshold values for the DNC experiment. Numbers in figure indicate slope threshold $T$. Morlet wavelet of wavenumber (a) $K_{0}=5$, (b) $K_{0}=7$, and (c) $K_{0}=9$. 
lution of high wavenumbers at the expense of greater uncertainty in locating events in space. The structural change in the atom used in the analysis has consequences for the estimation of $\Lambda_{T}(k)$. Steep wave events are detected from the peak value of the wavelet transform exceeding a set threshold. With the Morlet wavelet of wavenumber $K_{0}=9$, waves must be coherent over a longer distance in order to be detected as an event, compared to the analysis with a Morlet wavelet of lower wavenumber. Thus, it is expected that the overall amount of very steep wave events detected should decrease with increasing $K_{0}$ values for the same wave slope threshold. This is indeed exhibited in Fig. 9. However, even if a different $K_{0}$ is chosen, all characteristics of the results discussed earlier remain qualitatively the same, that is, the steep wave statistic decays exponentially with the slope squared and shows a moderately strong correlation with the degree of saturation. A more detailed sensitivity study is found in Scott (2003).

The $95 \%$ confidence limits for $\Lambda_{c T}(k)$ are plotted in Fig. 10. The steep wave statistic was assumed to be normally distributed with the error bars at a single wave slope threshold expressed as

$$
\left[\left(\bar{\Lambda}_{T}-\frac{s_{\Lambda} \times t_{M-1, \alpha / 2}}{\sqrt{M}}\right) \leq \Lambda_{T} \leq\left(\bar{\Lambda}_{T}+\frac{s_{\Lambda} \times t_{M-1, \alpha / 2}}{\sqrt{M}}\right)\right] .
$$

The mean steep wave statistic over the set of observations is designated as $\bar{\Lambda}_{T}$, where $M$ is the number of samples of the steep wave statistic, $s_{\Lambda}$ is the square root of the sample variance, and $\Lambda_{T}$ is the true steep wave statistic. The value $t_{M-1, \alpha / 2}$, obtained from the Student's $t$ distribution table, is the value such that

$$
\operatorname{prob}\left[t_{M-1}>t_{M-1, \alpha / 2}\right]=\frac{\alpha}{2},
$$

where $\alpha=0.05$ is the area under the Student's $t$ distribution curve. The interval is the symmetric interval with $2.5 \%$ on each side of the curve. The error bars are tighter at low wave slope thresholds than at high wave slope thresholds because of the decrease in variance in the signal.

\section{Conclusions}

The wavelet analysis methodology presented here is able to detect steep wave events and give estimates of the amount of high wave slope events that cover a given area of ocean. Analysis of the results shows that high wave slope crests appear over the entire range of wavenumbers resolved, with a large amount being much shorter in wavelength than the dominant wave. At low wave slope thresholds, the total crest length is approximately independent of wind forcing for all wave fields considered. The steep wave statistic $\Lambda_{c T}(k)$ then decays
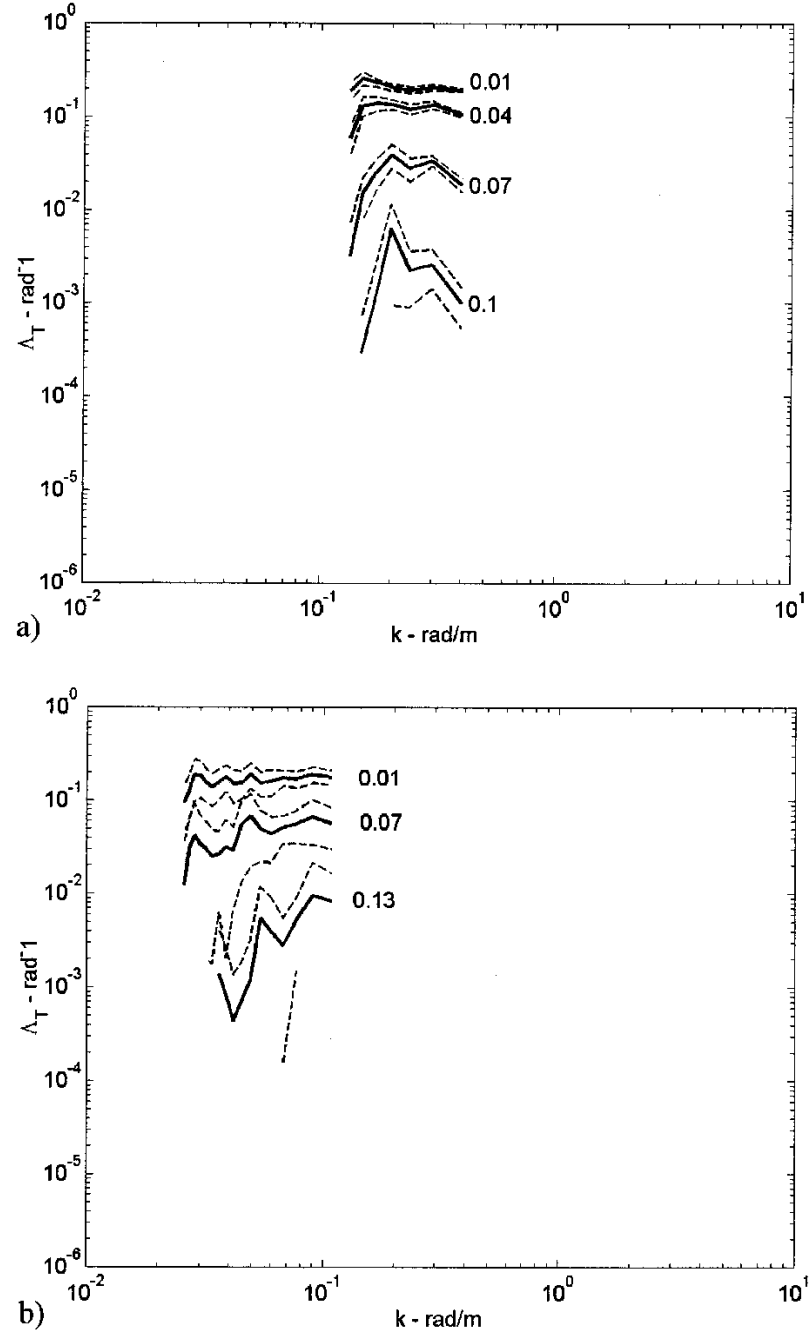

FIg. 10. The steep wave statistic $\Lambda_{T}(k)$ vs $k$ for different wave slope thresholds (indicated by the numbers in the plots). Dashed lines are $95 \%$ confidence limits. (a) DNC experiment and (b) 10 Jun SOWEX.

exponentially with the square of the slope threshold $T$. The exponent $p$ of the exponential decay is smaller for higher winds, yielding a larger number of very steep wave events.

If the steep wave statistic is hypothesized to evolve into the breaking wave statistic at a specific wave slope threshold, comparison of $\Lambda_{c T}(k)$ with previous independent measurements of the breaking wave statistic gives a wave slope threshold of about 0.12 . This threshold is consistent with the results of the numerical studies of Dold and Peregrine (1986). Comparison of the steep wave statistic at this extrapolated wave slope threshold with independent breaking wave measurements suggests that the steep wave statistic does not scale with the cube of the wind speed with other factors besides the wind speed affecting its level. Finally, $\Lambda_{c T}(k)$ at moderately large wave slope threshold correlates with the saturation spectrum $B(k)$ reasonably well. 
Acknowledgments. We would like to acknowledge Dr. Michael Banner for providing the SOWEX atmospheric data, and Drs. Stephen Belcher and David Wang for their helpful comments. This research was supported by the National Science Foundation Grant OCE0002314. Partial support was also provided by the Naval Research Laboratory under Grant N00173021G982 and the Office of Naval Research under Grant N00014-0110125.

\section{APPENDIX}

\section{Preservation of Wave Slope under Wavelet Transform}

Two signals whose wave slopes, $S=A \times k$ (where $A$ is amplitude and $k$ is wavenumber), are equal will have equal peak values of the wavelet transform $W f(a, s)$ at each of the signals' respective scales. To illustrate how this transform preserves the local average wave slope of a signal, consider two sinusoidal signals,

$$
\begin{aligned}
& f_{1}(u)=A \cos (k u) \\
& f_{2}(u)=\frac{A}{m} \cos (m k u),
\end{aligned}
$$

where $m$ is a positive constant. These signals have wave slopes $W f_{1}(a, 0)$ and $W f_{2}(\tilde{a}, 0)$, respectively, which are equal. Here $a$ and $\tilde{a}=a / m$ are the scales of the respective signals corresponding to wavenumbers, $k$ and $m k$.

The wavelet transform of $f_{1}(u)$ is

$$
\begin{aligned}
W f_{1}(a, s) & =\int_{-\infty}^{\infty} f_{1}(u) \frac{1}{a^{2}} \Psi\left(\frac{u-s}{a}\right) d u \\
& =\int_{-\infty}^{\infty} A \cos (k u) \frac{1}{a} \Psi\left(\frac{u-s}{a}\right) \frac{1}{a} d u .
\end{aligned}
$$

Consider the wavelet transform at zero translations, $s=$ 0 . Then

$$
W f_{1}(a, 0)=\int_{-\infty}^{\infty} A \cos (k u) \frac{1}{a} \Psi\left(\frac{u}{a}\right) \frac{1}{a} d u .
$$

Let us introduce the following change of variables,

$$
u=m \tilde{u} \quad d u=m d \tilde{u},
$$

which moves the signal and wavelet to another scale. Then

$$
W f_{1}(a, 0)=\int_{-\infty}^{\infty} A \cos (k m u) \frac{1}{a} \Psi\left(\frac{m u}{a}\right) \frac{m}{a} d u,
$$

where the tildes have been dropped. This equation can be rewritten as

$$
\begin{aligned}
W f_{1}(a, 0) & =\int_{-\infty}^{\infty} \frac{A}{m} \cos (k m u) \frac{1}{a / m} \Psi\left(\frac{u}{a / m}\right) \frac{1}{a / m} d u \\
& =\int_{-\infty}^{\infty} \frac{A}{m} \cos (m k \cdot u) \frac{1}{\tilde{a}} \Psi\left(\frac{u}{\tilde{a}}\right) \frac{1}{\tilde{a}} d u \\
& =\int_{-\infty}^{\infty} \frac{A}{m} \cos (m k \cdot u) \frac{1}{\tilde{a}^{2}} \Psi\left(\frac{u}{\tilde{a}}\right) d u \\
& =\int_{-\infty}^{\infty} f_{2}(u) \frac{1}{\tilde{a}^{2}} \Psi\left(\frac{u}{\tilde{a}}\right) d u=W f_{2}(\tilde{a}, 0),
\end{aligned}
$$

where $\tilde{a}=a / m$. Hence, the peak values of the transform are identical for the two signals.

\section{REFERENCES}

Banner, M. L., and X. Tian, 1998: On the determination of the onset of breaking for modulating surface gravity water waves. J. Fluid Mech., 367, 107-137.

- W. Chen, E. J. Walsh, J. B. Jensen, S. Lee, and C. Fandry, 1999: The Southern Ocean Waves Experiment. Part I: Overview and mean results. J. Phys. Oceanogr., 29, 2130-2145.

_ A. V. Babanin, and I. R. Young, 2000: Breaking probability for dominant waves on the sea surface. J. Phys. Oceanogr., 30, 3145-3160.

— J. R. Gemmrich, and D. Farmer, 2002: Multiscale measurements of ocean wave breaking probability. J. Phys. Oceanogr., 32, 3364-3375.

Cartwright, D. E., and M. S. Longuet-Higgins, 1956: The statistical distribution of the maxima of a random function. Proc. Roy. Soc. London, A237, 212-232.

Ding, L., and D. M. Farmer, 1994: Observations of breaking surface wave statistics. J. Phys. Oceanogr., 24, 1368-1387.

Dold, J. W., and D. H. Peregrine, 1986: Water-wave modulation. Proc. 20th Int. Conf. on Coastal Engineering, Taipei, Taiwan, ASCE, 163-175.

Gemmrich, J. R., and D. Farmer, 1999: Observations of the scale and occurrence of breaking surface waves. J. Phys. Oceanogr., 29, 2595-2606.

Hwang, P. A., D. W. Wang, E. J. Walsh, W. B. Krabill, and R. N. Swift, 2000: Airborne measurements of the wavenumber spectra of ocean surface waves. Part I: Spectral slope and dimensionless spectral coefficient. J. Phys. Oceanogr., 30, 2753-2767.

Liu, P., 1994: Wavelet spectrum analysis and ocean wind waves. Wavelets in Geophysics, E. Foufoula-Georgiou and P. Kumar, Eds., Academic Press, 151-166.

Melville, W. K., and P. Matusov, 2002: Distribution of breaking waves at the ocean surface. Nature, 417, 58-63.

Phillips, O. M., 1985: Spectral and statistical properties of the equilibrium range in wind-generated gravity waves. J. Fluid Mech., 156, 505-531.

, F. L. Posner, and J. P. Hansen, 2001: High range resolution radar measurements of the speed distribution of breaking events in wind-generated ocean waves: Surface impulse and wave energy dissipation rates. J. Phys. Oceanogr., 31, 450-460.

Scott, N. V., 2003: Observations of the wind-wave spectrum and steep wave statistics in open ocean waters. Ph.D. thesis, Graduate School of Oceanography, University of Rhode Island, $238 \mathrm{pp}$.

_ - T. Hara, P. A. Hwang, and E. J. Walsh, 2005: Directionality and crest length statistics of steep waves in open ocean waters. J. Atmos. Oceanic Technol., 22, 272-281. 